\title{
Un estudio de reclamos hospitalarios: el rol de la relación médico-paciente
}

\author{
PHILIPPA MOORE ${ }^{1,4}$, ALEX VARGAS ${ }^{2,3}$, \\ SALOMÓN NÚÑEZ ${ }^{\text {a }}$ STEFANO MACCHIAVELLO ${ }^{a}$
}

Departamento de Medicina Familiar. ${ }^{2}$ Departamento de Cirugía Oncológica y Máxilofacial. 3unidad de Comunicación y Relación en Atención de Salud, Escuela de Medicina Pontificia Universidad Católica de Chile.

aAlumno de Medicina de la Pontificia Universidad Católica de Chile.

Fuente de Apoyo financiero: Ninguno

Recibido el 14 de enero de 2011, aceptado el 6 de mayo de 2011

Correspondencia a:

Dra. Philippa Moore Departamento de Medicina Familiar, Lira 44, Santiago, Chile.

Fax 5186760 E-mail: moore@med.puc.cl

\section{A study of hospital complaints and the role of the doctor-patient communication}

Background: Medical practice in Chile has changed dramatically over the last few years. Patients have raised their expectations and there is a growing number of complaints, and malpractice lawsuits. The doctor-patient relationship plays a fundamental role in patient satisfaction and has also been identified as an issue in most medical lawsuits. Aim: To analyze the importance of doctor-patient communication in the complaints received in a university hospital in Chile. Material and Methods: Review of all complaints received at the office for quality of care at a university hospital. Complaints classified in delay, manners and information categories were selected for further analysis. Results: Of a total of 8931 complaints registered between 2001 and 2008, 635 (19\%) involved a doctor. Fifty one per cent of the latter, were related to the doctor-patient relationship. Of these, 146 cases (45\%) were further classified as "Dysfunctional delivery of Information", 74 cases (23\%) as "Not understanding the patient/family's perspective", 54 cases (17\%) as "Discrediting the patient or family's views" and 49 cases (15\%) as "Lack of communication". Conclusions: The percentage of complaints related to communication with the doctor is high, though lower than cited in other studies. The most common complaint is the dysfunctional delivery of information.

(Rev Med Chile 2011; 139: 880-885).

Key words: Communication; Patient satisfaction; Physician-patient relations; Professional impairment.
E 1 ejercicio de la medicina en Chile ha cambiado notoriamente en los últimos años ${ }^{1}$. La satisfacción de los pacientes con la relación establecida con su médico tratante ha cambiado ${ }^{2}$. Los pacientes tienen mayores expectativas y hay cifras crecientes de querellas y demandas ${ }^{3}$. Se estima que alrededor de 1 de cada 10 personas hospitalizadas sufre un evento adverso de los cuáles $50 \%$ son prevenibles. Pero no todo efecto adverso resulta en un reclamo y a veces hay reclamos donde no hubo eventos adversos. Hay una relación entre la satisfacción del paciente, el número de reclamos y la incidencia de litigios y demandas por mal praxis. Diversos estudios, incluyendo estudios chilenos ${ }^{4,5}$ analizan los factores que más influyen en la satis- facción de los pacientes y cuáles son los predictores de reclamos, querellas y juicios médicos ${ }^{6-8}$.

\section{¿Cuál es el rol de la comunicación médico-} paciente en relación a reclamos y juicios?

La relación médico-paciente tiene un rol fundamental en el cuidado médico. Además de estar asociada con decisiones médicas más apropiadas, mejor adherencia a los tratamientos y mejores resultados terapéuticos, una comunicación médicopaciente efectiva está relacionada con una mayor satisfacción tanto de los pacientes como de los médicos9. Varios estudios han encontrado una fuerte relación entre la forma de comunicación del médico y las demandas legales. El clásico estudio 
Reclamos hospitalarios y relación médico-paciente - P. Moore et al

de Beckman, cuya categorización es citada frecuentemente por la literatura, encontró que 70\% de los juicios médicos estaban relacionados con problemas de comunicación médico-paciente ${ }^{10}$. Más recientemente, Levinson y col en un estudio comparativo de la relación entre la calidad de la comunicación de médicos de atención primaria (internistas y médicos familiares) o cirujanos (generales y traumatólogos) con sus pacientes y litigios médicos, demostró que la comunicación médico-paciente tenía más importancia en los litigios contra médicos de atención primaria que contra los cirujanos, cuyos litigios estaban más relacionados con efectos adversos de sus resultados quirúrgicos ${ }^{11}$. Estudios sobre reclamos son escasos, ninguno realizado en Chile. La mayoría de los reclamos (70\%) también está ligado a problemas de comunicación de algún miembro del equipo de salud $^{12}$. La mayoría de los reclamos relacionados con comunicación son válidos: en un estudio de reclamos revisado por peer review, 78\% de los reclamos relacionados con comunicación fueron aceptados como válidos, comparado con $25 \%$ de los reclamos por fallas en el tratamiento y $20 \%$ por errores clínicos ${ }^{13}$. En resumen, comunicación efectiva no es ninguna garantía contra litigios o demandas, pero puede jugar un rol decisivo para disminuir el número de reclamos y aumentar la satisfacción de los pacientes ${ }^{14}$.

\section{¿Que tipo de comunicación está asociada a reclamos, litigios y decisiones judiciales que asignan responsabilidad médica?}

En la Tabla 1 se resumen las características de los médicos que reciben más demandas ${ }^{11}$. Existe mayor cantidad de demandas contra cirujanos con tonos de voz autoritaria (entendiendo por ésta aquella más sonora, más profunda, moderadamente rápida, no acentuada y vocalizada de manera claramente articulada $)^{15}$. El momento en que el médico le daba las explicaciones a su paciente (antes o después de instaurar un tratamiento) y la forma en que lo hacía también son importantes: explicaciones relevantes y específicas dadas antes del tratamiento o la cirugía por más de dos médicos y en las cuales se hace participar activamente a la familia son más aceptables ${ }^{16}$.

Uno de los estudios más citado es el estudio de Beckman. A través de un proceso de validación consensuado se creó un marco para analizar los problemas de relación médico-paciente en re- clamos y demandas. Surgieron 4 categorías, dos de las cuales son definidas como comunicación fallida: "Entrega disfuncional de información" (no entregar una explicación, no mantener al paciente y/o familiares informados, culpar al paciente y/o familia por un mal desenlace o insensibilidad al informar el paciente); y, "Desvalorización de la opinión del paciente y/o familiares" (ignorar, negar, desvalorizar o no escuchar la opinión del paciente y/o familiar o los síntomas/sufrimiento del paciente). Las otras dos categorías son "Falta de comprensión del paciente $y / 0$ familiares" (no solicitar la opinión del paciente y/o familiares, no reconocer el impacto psico-social y no considerar el malestar del paciente) y "Deserción" (un rango de temas que van desde la percepción del paciente que el médico no está disponible, el médico manda un reemplazante por considerarse demasiado importante para conversar directamente con el paciente, hasta un sensación de abandono o rechazo por parte del paciente).

El objetivo del trabajo aquí presentado fue describir y analizar el rol de la relación médicopaciente en los reclamos recogidos con los instrumentos de que dispone actualmente el Hospital Clínico de la Pontificia Universidad Católica de Chile en su Programa de Mejoramiento Continuo.

\section{Material y Método}

Se realizó un estudio descriptivo, retrospectivo, a través del cual se analizaron los registros de

\section{Tabla 1. Errores en las estrategias comunicacio-} nales de médicos que reciben demandas ${ }^{11}$

- Tiempo de entrevista más corta (15 minutos versus 18,3 minutos)

- Menos uso de:

- Habilidades comunicacionales que crean estructura a la entrevista

- Escucha Activa

- Facilitación-frases que alientan al paciente a contar su historia

Preguntas acerca de la opinión del paciente sobre plan de manejo

- Menos humor durante la entrevista 
reclamos canalizados por el Programa de Mejoramiento Continuo de la Dirección de Dependencias Docentes Asistenciales (DDDA), actualmente centralizados en la Dirección de Calidad y Seguridad Asistencial (DICSA), entre el $1^{\circ}$ de enero de 2001 y el 31 de diciembre de 2008. El sistema de análisis de la DICSA facilitó esta base de datos, con los reclamos ya ordenados y clasificados en ocho categorías: 1) Información; 2) Trato; 3) Demora; 4) Seguridad Asistencial; 5) Facturación; 6) Pérdida de documentos clínicos; 7) Infraestructura y 8) Otros); cada una con subcategorías.

Se seleccionaron las tres categorías relevantes a este estudio (Demora, Trato e Información), cada una con sus subcategorías; y se extractaron los reclamos que cumplieron nuestros criterios de inclusión. Cada una de los reclamos hallados en estas subcategorías fue clasificada por los investigadores como " $\mathrm{C}$ " (problemas médicos y comunicacionales), " $\mathrm{N}$ " (problemas médicos no comunicacionales), "B" (problemas no médicos) o "P" (probable problema comunicacional). Los reclamos clasificados como B o $\mathrm{P}$, fueron sometidos a un segundo análisis por dos investigadores, independientemente, reclasificándose en $\mathrm{C}, \mathrm{N}$ o B. Este último análisis arrojó un coeficiente kappa de 0,64 (bueno).

Las diferencias también fueron re-analizadas y consensuadas por ambos investigadores y las diferencias no consensuadas se llevaron al grupo completo de investigadores y se dirimió en consenso general.

El primer criterio de inclusión fue que el reclamo se haya generado debido al actuar médico directamente y no por el quehacer de otro profesional o algún otro miembro del equipo de salud. El segundo criterio de inclusión fue que el reclamo involucrara aspectos comunicacionales con el paciente, excluyéndose aquellos reclamos debidos a problemas de otro orden, no comunicacional, tales como pérdida de exámenes, disconformidad en la cuenta final, disconformidad con las acomodaciones hospitalarias, etc.

Con la misma metodología de pares y consensos grupales, todos los reclamos que cumplieron con estos dos criterios de inclusión fueron reclasificados de acuerdo a los criterios de Beckman ${ }^{10}$, en cuatro grupos: 1) "Entrega disfuncional de información"; 2) "Falta de comprensión del paciente o familiares"; 3) "Desvalorización de la opinión del paciente o familiares" y 4) "Comunicación Ausente".

\section{Resultados}

Se recibieron un total de 8.931 reclamos en las oficinas de la Dirección de Calidad y Seguridad Asistencial (DICSA) del Hospital Clínico de la Pontificia Universidad Católica de Chile, en el período entre enero de 2001 y diciembre de 2008. De estos reclamos, 635 (7,1\% del total de la muestra) cumplían con el primer criterio de inclusión: reclamos relacionados con el actuar médico. De estos, 323 (51\% de los reclamos que involucraron a un médico) cumplieron el segundo criterio de inclusión: estar relacionados con aspectos comunicacionales del paciente (Tabla 2).

Los reclamos médicos comunicacionales así obtenidos, reclasificados en las cuatro categorías de Beckman, se distribuyeron de la siguiente forma: "Entrega disfuncional de información (Falta de Información)" registró 146 casos (45\%), seguido de "Falta de Comprensión" con 74 casos (23\%); luego "Desacreditación" con 54 casos (17\%), y, finalmente "Comunicación Ausente" con 49 casos (15\%) (Tabla 3).

\section{Discusión}

Aunque nuestra base de datos muestra una clara tendencia en el aumento de reclamos (Figura 1 , con el incremento sostenido de las cifras entre 2001 y 2008), Chile es un país donde el porcentaje de reclamos contra el médico todavía es baja en

\section{Tabla 2. Distribución de la muestra de reclamos registrada en el período 2001-2008}

\begin{tabular}{|lrr|}
\hline & n & \% \\
\hline Total de reclamos 2001 - 2008 & 8.931 & \\
\hline $\begin{array}{l}\text { Primer criterio de inclusión: } \\
\text { Reclamos relacionados c/ el acto médico }\end{array}$ & & \\
- Relacionados con el actuar médico & 635 & 7 \\
- No relacionados con el actuar médico & 8.296 & 93 \\
$\begin{array}{l}\text { Segundo criterio de inclusión: } \\
\text { Reclamos de relación médico-paciente }\end{array}$ & & \\
- Relacionados con la relación médico- & 323 & 51 \\
paciente & & \\
- No relacionados con la relación médico & 312 & 49 \\
\hline & & \\
\hline
\end{tabular}


Reclamos hospitalarios y relación médico-paciente - P. Moore et al

Tabla 3. Comparación de la muestra de reclamos obtenida según categorías de Beckman

\begin{tabular}{|lcccc|}
\hline Categorías & $\begin{array}{c}\text { Nuestro estudio } \\
\text { (Reclamos) }\end{array}$ & $\begin{array}{c}\text { Estudio Beckman } \\
\text { (Juicios) }\end{array}$ \\
\hline Entrega disfuncional de información & 146 & $45 \%$ & 5 & $13 \%$ \\
\hline Falta de comprensión (con paciente y/o familiares) & 74 & $23 \%$ & 10 & $26 \%$ \\
\hline Desacreditación (desvalorización) & 54 & $17 \%$ & 11 & $29 \%$ \\
\hline Deserción (comunicación ausente) & 49 & $15 \%$ & 12 & $32 \%$ \\
\hline Total de reclamos relacionados con la relación médico-paciente & 323 & $100 \%$ & 38 & $100 \%$ \\
\hline
\end{tabular}

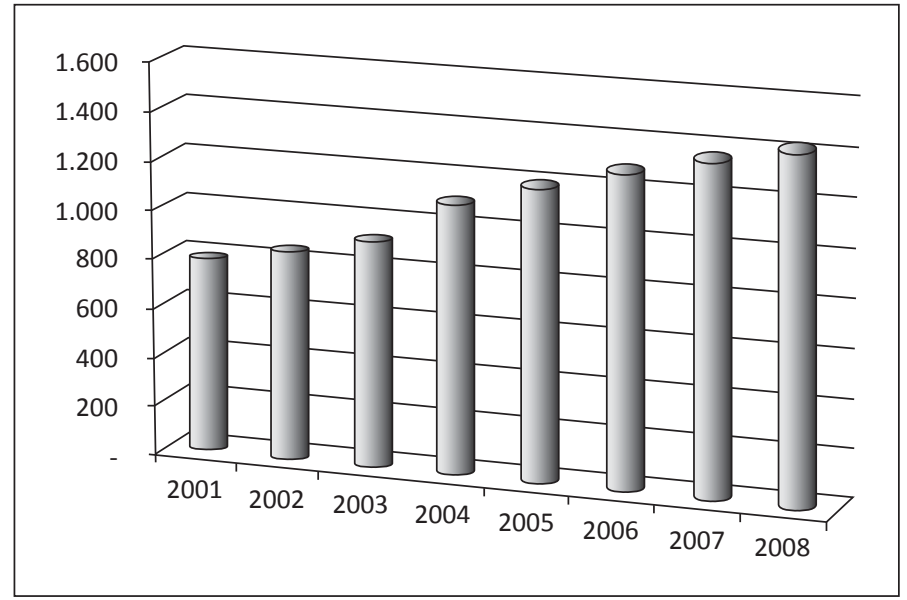

Figura 1. Reclamos totales recibidos en el Hospital Clínico de la PUC (Período 2001-2008). comparación con países como EE.UU. de Norteamérica en lo cual se estima que cada año $25 \%$ de los médicos clínicos reciben una querella. Entre los pocos reclamos contra el médico, es interesante destacar que la mitad de estos (51\%) estuvieron relacionados con la comunicación médico paciente, cifra considerable pero más bajo que lo encontrado en estudios en otros países ${ }^{12,17}$, en los cuales se han reportado entre 80 y $90 \%$, debido probablemente a diferencias culturales ${ }^{18}$.

Categorizar los reclamos relacionados con comunicación médico-paciente con el marco descrito por Beckman nos permite comparar nuestros reclamos relacionados con la relación médico-paciente con otros estudios. Los porcentajes obtenidos en cada categoría en nuestro análisis difieren de los encontrados en el estudio original de Beckman, quizás porque el estudio original no fue de reclamos sino de juicios médicos ${ }^{10}$. Los juicios afectan solamente a un porcentaje pequeño de los médicos y son considerados eventos poco frecuentes, mientras el número de reclamos es significativamente mayor. ${ }^{16,19}$ Sin embargo, estudios de los reclamos puede dar luz a la prevención de demandas médicas, ya que se ha encontrado una relación directa entre el número de reclamos que tenga un médico y su riesgo de tener una demanda; aunque se reconoce que se requieren estudios con gran número de casos de juicios para identificar con seguridad esta relación ${ }^{8}$. Esto implica que médicos con altas tasas de reclamos pueden estar expuestos a mayor riesgo de demandas que sus colegas de la misma especialidad con menores tasas de reclamos. En el estudio original de Beckman se encontró que el problema comunicacional más frecuente en los juicios contra médicos fue de la categoría de "Deserción". Es posible que los reclamos relacionados con esta categoría en nuestro estudio tengan mayor riesgo de terminar en una demanda contra el médico.

Cuarenta y cinco por ciento de los reclamos de nuestro estudio, relacionados con comunicación 
médico-paciente, correspondieron a la categoría "Entrega disfuncional de la información". Es probable que este alto porcentaje, bastante superior al obtenido por Beckman, se deba a que la enseñanza del enfoque centrado en el paciente -donde el médico toma en cuenta la agenda del paciente con sus emociones, preocupaciones y expectativas-, es aún relativamente nuevo en Chile ${ }^{20}$.

La base de datos original tiene una categoría "Trato" con un sub-categoría "Trato médico" A primera vista se podría pensar que esta sub-categoría representaba todos los reclamos relacionados con comunicación médico-paciente; sin embargo, nuestro análisis demuestra que la sub-categoría "Trato médico" contiene solamente el 10\% de los reclamos relacionados con la comunicación médico-paciente, y tal como se ha descrito, muchos de los reclamos relacionado con la comunicación médico-paciente están en relación con la entrega de información.

Si queremos reducir el número de reclamos -y por lo tanto, el riesgo de juicios-, las instituciones de salud debieran tener estrategias tanto para mejorar la calidad de su servicio, como para apoyar a los médicos que hayan generado reclamos ${ }^{21}$. La experiencia de tener un reclamo en contra puede ser devastadora para un médico; los que reciben reclamos se sienten sin apoyo y temen las consecuencias $^{22,23}$. Por lo tanto, cualquier estrategia debe incluir ayuda para confrontar sus fallas y mejorar sus habilidades o actitudes ${ }^{24}$. Un estudio reciente demuestra que médicos con un desempeño subóptimo pueden ser precozmente identificados y eventualmente recibir capacitación remedial. Tamblyn y colaboradores siguieron cohortes de médicos recién egresados por entre 2 y 12 años y demostraron que las notas obtenidas sobre habilidades comunicacionales en el examen médico nacional de Canadá son predictores de las tasas de reclamos de sus futuros profesionales ${ }^{25}$.

La mayoría de los médicos tiene su primera experiencia con error médico durante su formación. Es importante que sus docentes le ayudan al estudiante para que su aprendizaje sobre esta experiencia puede ser constructiva, dado que esta experiencia negativa puede durar el resto de su carrera $^{26}$. También es importante observar y evaluar las habilidades comunicacionales de nuestros estudiantes y médicos; y ofrecer capacitación remedial a los que tienen habilidades sub-óptimas ${ }^{27}$. Cursos sobre comunicación médico-paciente pueden mejorar las habilidades comunicacionales de los médicos, independientemente de su edad o nivel de experiencia ${ }^{28}$; y también pueden mejorar la satisfacción de sus pacientes y disminuir sus tasas de reclamos $^{29,30}$. Las escuelas de medicina han respondido frente a esta evidencia incorporando la formación en la relación médico-paciente en su docencia y en sus procesos de evaluación ${ }^{20}$. En los últimos años la Escuela de Medicina de la Pontificia Universidad Católica de Chile ha incorporado cursos formales en habilidades comunicacionales e instancias de evaluación de estas a lo largo del currículo de pregrado y en algunos programas de post-grado. Además, ofrece cursos para los docentes clínicos sobre cómo enseñar sobre la comunicación médico-paciente.

Este estudio es uno de los primeros estudios en Chile sobre reclamos hospitalarios. Los resultados obtenidos pueden constituir la base diagnóstica para evaluar el impacto de los esfuerzos que esperamos se hagan por mejorar la relación médicopaciente sobre los reclamos de los pacientes.

Agradecimientos: Queremos expresar nuestros agradecimientos al Dr. Andrés Poblete y su equipo en la Dirección de Calidad y Seguridad Asistencial (DICSA), Pontificia Universidad Católica de Chile por facilitar el acceso a los datos.

\section{Referencias}

1. Jiménez de la JJ. [Changes in the environment of medical practice]. Rev Med Chile 2004; 132 (5): 637-42.

2. Bascuñán RM. [Changes in physician-patient relationship and medical satisfaction]. Rev Med Chile 2005; 133 (1): 11-6.

3. Pizarro WC. [Perspectives in medical liability]. Rev Med Chile 2008; 136 (4): 539-43.

4. Bozzo G, Martínez B, Sepúlveda P, Carvacho C, Rivera G, Moore P. Desarrollo y validación de una encuesta para evaluar la satisfacción de los pacientes con la consulta médica en consultorios de atención primaria. Rev Med Chile 1995; 123: 1160-4.

5. Castillo L, Dougnac A, Vicente I, Muñoz V, Rojas V. [Predictors of the level of patient satisfaction in a university hospital]. Rev Med Chile 2007; 135 (6): 696-701.

6. Stelfox HT, Gandhi TK, Orav EJ, Gustafson ML. The relation of patient satisfaction with complaints against physicians and malpractice lawsuits. Am J Med 2005; 118 (10): 1126-33.

7. Mazor KM, Reed GW, Yood RA, Fischer MA, Baril J, 
Gurwitz JH. Disclosure of medical errors: what factors influence how patients respond? J Gen Intern Med 2006; 21 (7): 704-10.

8. Hickson GB, Federspiel CF, Pichert JW, Miller CS, Gauld-Jaeger J, Bost P. Patient complaints and malpractice risk. JAMA 2002; 287 (22): 2951-7.

9. Silverman J, Kurtz SM, Draper J. Skills for Communicating with Patients. Second ed. Abingdon: Radcliffe Medical Press; 2005.

10. Beckman HB, Markakis KM, Suchman AL, Frankel RM. The doctor-patient relationship and malpractice. Lessons from plaintiff depositions. Arch Intern Med 1994; 154 (12): 1365-70.

11. Levinson W, Roter DL, Mullooly JP, Dull VT, Frankel RM. Physician-patient communication. The relationship with malpractice claims among primary care physicians and surgeons. JAMA 1997; 19; 277(7): 553-9.

12. Cave J, Dacre J. Dealing with complaints. BMJ 2008; 336 (7639): 326-8.

13. Donaldson LJ, Cavanagh J. Clinical complaints and their handling: a time for change? Qual Health Care 1992; 1 (1): 21-5.

14. Lussier MT, Richard C. Doctor-patient communication: complaints and legal actions. Can Fam Physician 2005; 51: 37-9: 37-9.

15. Ambady N, Laplante D, Nguyen T, Rosenthal R, Chaumeton N, Levinson W. Surgeons' tone of voice: a clue to malpractice history. Surgery 2002; 132 (1): 5-9.

16. Hamasaki T, Takehara T, Hagihara A. Physicians' communication skills with patients and legal liability in decided medical malpractice litigation cases in Japan. BMC Fam Pract 2008; 9: 43.

17. Kinnersley P, Edwards A. Complaints against doctors. BMJ 2008; 336 (19): 841-2.

18. Wissow LS. Communication and malpractice claims-where are we now? Patient Educ Couns 2004; 52 (1): 3-5.

19. Rodríguez HP, Rodday AM, Marshall RE, Nelson KL,
Rogers WH, Safran DG. Relation of patients' experiences with individual physicians to malpractice risk. Int J Qual Health Care 2008; 20 (1): 5-12.

20. Rosselot E. [Looking for the physicians that our countries need: emphasis on communication and training of academics]. Rev Med Chile 2003; 131 (3): 331-7.

21. Mena P. Error Médica y eventos adversos. Rev Chil Pediatr 2008; 79 (3): 319-26.

22. Jain A, Ogden J. General practitioners' experiences of patients' complaints: qualitative study. BMJ 1999; 318 (7198): 1596-9.

23. Cunningham $\mathrm{W}$. The immediate and long-term impact on New Zealand doctors who receive patient complaints. N Z Med J 2004; 117 (1198): U972.

24. Baker R. Learning from complaints about general practitioners. BMJ 1999; 318 (7198): 1567-8.

25. Tamblyn R, Abrahamowicz M, Dauphinee D, Wenghofer E, Jacques A, Klass D, et al. Physician scores on a national clinical skills examination as predictors of complaints to medical regulatory authorities. JAMA 2007; 298 (9): 993-1001.

26. Mazor KM, Fischer MA, Haley HL, Hatem D, Rogers HJ, Quirk ME. Factors influencing preceptors' responses to medical errors: a factorial survey. Acad Med 2005; 80 (10 Suppl): S88-S92.

27. Makoul G, Curry RH. The value of assessing and addressing communication skills. JAMA 2007; 298 (9): 1057-9.

28. Fellowes D, Wilkinson S, Moore P. Communication skills training for health care professionals working with cancer patients, their families and/or carers. Cochrane Database Syst Rev 2003; 2: CD003751.

29. Peskin T, Micklitsch C, Quirk M, Sims H, Primack W. Malpractice, patient satisfaction, and physician-patient communication. JAMA 1995; 274 (1): 22.

30. Lau FL. Can communication skills workshops for emergency department doctors improve patient satisfaction? J Accid Emerg Med 2000; 17 (4): 251-3. 